\title{
Unconventional dc Transport in Rashba Electron Gases
}

\author{
Valentina Brosco, ${ }^{1}$ Lara Benfatto, ${ }^{1}$ Emmanuele Cappelluti, ${ }^{1}$ and Claudio Grimaldi ${ }^{2}$ \\ ${ }^{1}$ ISC-CNR and Department of Physics, Sapienza University of Rome, Piazzale Aldo Moro 2, 00185 Rome, Italy \\ ${ }^{2}$ Laboratory of Physics of Complex Matter, Ecole Polytechnique Fédérale de Lausanne, \\ Station 3, CH-1015 Lausanne, Switzerland
}

(Received 27 November 2015; published 20 April 2016)

\begin{abstract}
We discuss the transport properties of a disordered two-dimensional electron gas with strong Rashba spin-orbit coupling. We show that in the high-density regime where the Fermi energy overcomes the energy associated with spin-orbit coupling, dc transport is accurately described by a standard Drude's law, due to a nontrivial compensation between the suppression of backscattering and the relativistic correction to the quasiparticle velocity. On the contrary, when the system enters the opposite dominant spin-orbit regime, Drude's paradigm breaks down and the dc conductivity becomes strongly sensitive to the spin-orbit coupling strength, providing a suitable tool to test the entanglement between spin and charge degrees of freedom in these systems.
\end{abstract}

DOI: 10.1103/PhysRevLett.116.166602

Spin-orbit (SO) coupling is a fundamental ingredient in spintronics [1], as it provides an advantageous locking between spin and electron orbital momentum. Recently, intense research efforts [2] have been devoted to twodimensional materials with broken inversion symmetry, where the SO strength, parametrized by a characteristic energy scale $E_{0}$, can be tuned by means of external conditions (electric fields, gating, doping, pressure, strain, etc.). In most of these systems (for example, surface alloys [3-9], layered bismuth tellurohalides [10-16], HgTe quantum wells [17], and interfaces between complex oxides [18-32]) the total charge carrier density $n$ can be tuned down to very small concentrations, implying very small Fermi energies $E_{F}$. Although the high-density (HD) regime $E_{F} \gtrsim E_{0}$ has been widely investigated [2,33-38], relatively less attention has been paid to the opposite regime of dominant SO (DSO), $E_{0} \gtrsim E_{F}$.

In this Letter we provide a detailed investigation of the $\mathrm{dc}$ conductivity of a 2D electron gas (2DEG) with Rashba [39] SO coupling in the different density regimes. Using a Boltzmann approach and a fully quantum analysis based on the Kubo formula, we show that in the high-density regime $E_{F} \gtrsim E_{0} \mathrm{dc}$ transport is independent of the SO strength, and the dc conductivity $\sigma_{\mathrm{dc}}$ of electrons having effective mass $m$ and scattering time $\tau_{0}$ follows the conventional Drude law for 2DEGs,

$$
\sigma_{\text {Drude }}=\frac{n e^{2} \tau_{0}}{m}
$$

which results from a nontrivial cancellation of the SO coupling effects on the quasiparticle velocity and transport scattering time. Remarkably, as soon as the system enters the DSO regime $E_{0} \gtrsim E_{F}$, Drude's paradigm Eq. (1) breaks down and the dc conductivity accurately follows the analytical formula:

$$
\sigma_{\mathrm{DSO}}=\frac{e^{2} \tau_{0} n_{0}}{2 m}\left(\frac{n^{4}}{n_{0}^{4}}+\frac{n^{2}}{n_{0}^{2}}\right), \quad n \leq n_{0},
$$

where $n_{0}=2 m E_{0} /\left(\pi \hbar^{2}\right)$ is the density at $E_{F}=E_{0}$. In contrast to the linear dependence of $\sigma_{\mathrm{dc}}$ on the charge density found in the HD regime, $n \geq n_{0}$, Eq. (2) predicts an unconventional nonlinear behavior of $\sigma_{\mathrm{dc}}$ with $n$ that is controlled by the SO interaction encoded in $n_{0}$. The relevance of this result is twofold: demonstrating that dc transport is strongly sensitive to Rashba SO coupling, not only does it suggest that SO coupling could be measured in a transport experiment, but also, which is more important for applications, it points to the possibility of tuning the conductivity of a $2 \mathrm{DEG}$ by tuning the $\mathrm{SO}$ coupling strength via external gates.

A disordered Rashba 2DEG confined to the $(x, y)$ plane is described by the following Hamiltonian:

$$
H=\int d \mathbf{r} \Psi^{\dagger}(\mathbf{r})\left[\frac{p^{2}}{2 m}+\alpha \hat{z} \cdot(\mathbf{p} \times \vec{\sigma})+V_{\text {imp }}(\mathbf{r})\right] \Psi(\mathbf{r}),
$$

where $V_{\mathrm{imp}}(\mathbf{r})$ is the disorder potential, $\alpha$ is the SO coupling, $\vec{\sigma}$ is the vector of Pauli matrices, and $\Psi(\mathbf{r})$ and $\Psi^{\dagger}(\mathbf{r})$ are spinor fields which, respectively, create and destroy electrons at position $\mathbf{r}$ and $\hat{a}=\vec{a} /|\vec{a}|$. Here we limit ourselves to the simplest case of Gaussian random disorder with "white noise" correlations; namely, we set $\left\langle V_{\text {imp }}(\mathbf{r}) V_{\text {imp }}\left(\mathbf{r}^{\prime}\right)\right\rangle_{\text {imp }}=n_{i} v_{\text {imp }}^{2} \delta\left(\mathbf{r}-\mathbf{r}^{\prime}\right)$, where $v_{\text {imp }}$ and $n_{i}$ denote, respectively, the scattering strength and the impurity density. In the absence of impurities, $H$ gives an electronic spectrum, depicted in Fig. 1, consisting of two bands with dispersion $E_{p}^{s}-E_{0}=\left(p+s p_{0}\right)^{2} /(2 m)-E_{0}$, where $p_{0}=m \alpha, E_{0}=m \alpha^{2} / 2$, and $s= \pm 1$ denotes the 


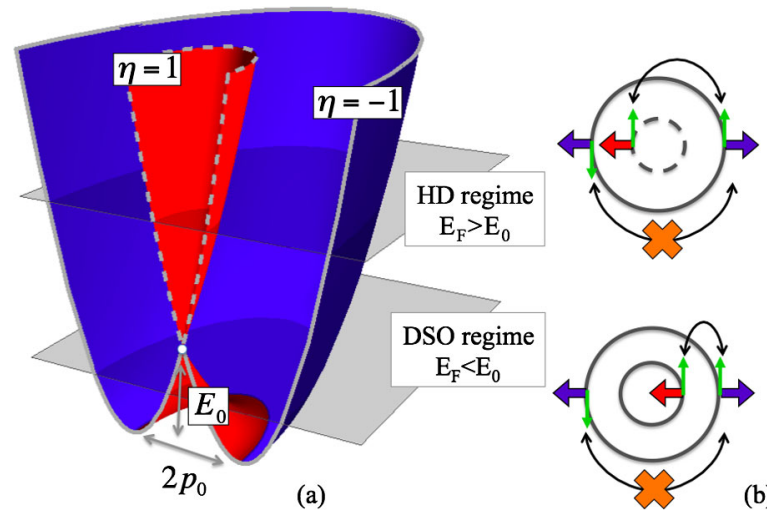

FIG. 1. (a) Dispersion of the Rashba model. The solid (dashed) contours and the red (blue) colors denote, respectively, $s$ and $\eta$. (b) Examples of allowed and forbidden backscattering processes. Thick (red and blue) and thin green arrows denote the quasiparticle velocity $\vec{v}_{\mathbf{p} s}$ and the spin $\langle\vec{\sigma}\rangle_{\mathbf{p} s}$ of each state, respectively.

eigenvalue of the helicity operator $S$ defined as usual as $S=\hat{z} \cdot(\hat{p} \times \vec{\sigma})$. In the following we measure the Fermi energy $E_{F}$ from the lower band edge, so that $E_{F}=E_{0}$ corresponds to the "Dirac point" $p=0$, and the HD and DSO regimes are realized, respectively, for $E_{F}>E_{0}$ and $E_{F}<E_{0}$.

As already noted in Ref. [40], the onset of the DSO regime leads to a rather sharp change in density of states (DOS) at the Fermi level. Indeed, while for $E_{F}>E_{0}$ the DOS is constant, $N\left(E_{F}\right)=N_{0}=m /\left(\pi \hbar^{2}\right)$, for $E_{F}<E_{0}$ it displays a van Hove singularity, $N\left(E_{F}\right)=N_{0} \sqrt{E_{0} / E_{F}}$, leading to a modification of the dependence of the density on the Fermi energy [40]:

$$
n \simeq \begin{cases}N_{0}\left(E_{F}+E_{0}\right) & E_{F}>E_{0} \\ 2 N_{0} \sqrt{E_{F} E_{0}} & E_{F}<E_{0} .\end{cases}
$$

In the presence of static diluted disorder, the singular behavior of the DOS reflects directly on the quasiparticle's lifetime that, using the Fermi golden rule (see, e.g., Ref. [41]), can be cast as $\tau\left(E_{p}^{s}\right)=\mathcal{V}\left[\sum_{\mathbf{p}^{\prime} s^{\prime}} Q_{\mathbf{p}^{\prime} s^{\prime}}^{\mathbf{p} s}\right]^{-1}$, where $\mathcal{V}$ is the 2D volume of the sample and

$$
Q_{\mathbf{p}^{\prime} s^{\prime}}^{\mathbf{p} s}=\pi n_{i} v_{\mathrm{imp}}^{2}\left(1+s s^{\prime} \hat{p} \cdot \hat{p}^{\prime}\right) \delta\left(E_{p}^{s}-E_{p^{\prime}}^{s^{\prime}}\right)
$$

is the scattering kernel. Using Eqs. (4) and (5) one can easily show that the quasiparticle scattering time scales linearly with the density in the DSO regime, i.e.,

$$
\tau=\tau\left(E_{F}\right)= \begin{cases}\tau_{0} & E_{F}>E_{0} \\ \tau_{0} \sqrt{E_{F} / E_{0}}=\tau_{0}\left(n / n_{0}\right) & E_{F}<E_{0},\end{cases}
$$

where $\tau_{0}=\hbar^{2} /\left(m n_{i} v_{\text {imp }}^{2}\right)$ denotes the quasiparticle scattering time in the absence of SO.
To explain the behavior of the conductivity across the different regimes, we start by recalling the definition of the velocity operator in the helicity basis,

$$
[\vec{v}]_{s s^{\prime}}=\vec{v}_{\mathbf{p} s} \delta_{s s^{\prime}}-i \alpha s\left(1-\delta_{s s^{\prime}}\right) \hat{t}_{p}
$$

where $\vec{v}_{\mathbf{p} s}=\nabla_{\mathbf{p}} E_{p}^{s}=\hat{p}(p / m+s \alpha)$ denotes the quasiparticle velocity and $\hat{t}_{p}$ is defined as $\hat{t}_{p}=\left\{p_{y} / p,-p_{x} / p\right\}$. An important thing to underline here, general for any chiral system, is that in the presence of SO coupling also the velocity acquires a spin structure, which has a deep impact on the transport properties. As we discuss in more detail below, to a first approximation the conductivity can be described within a standard semiclassical Boltzmann approach that only keeps the quasiparticle current, arising from the diagonal components of the velocity operator Eq. (7). In the relaxation time approximation at $T=0, \sigma_{\mathrm{dc}}$ can be then estimated as

$$
\sigma_{\mathrm{dc}} \simeq \sigma_{\mathrm{dc}}^{B}=\frac{e^{2}}{2 \mathcal{V}} \sum_{\mathbf{p} s} \delta\left(E_{F}-E_{p}^{s}\right)\left|\vec{v}_{\mathbf{p} s}\right|^{2} \tau_{p s}^{\mathrm{tr}}
$$

where the transport scattering times $\tau_{p s}^{\mathrm{tr}}$ satisfy the following equations [42]:

$$
\frac{\tau_{p s}^{\mathrm{tr}}}{\tau\left(E_{p}^{s}\right)}=1+\frac{1}{\mathcal{V}} \sum_{\mathbf{p}^{\prime} s^{\prime}} Q_{\mathbf{p} s}^{\mathbf{p}^{\prime} s^{\prime}} \frac{\vec{v}_{\mathbf{p}^{\prime} s^{\prime}} \cdot \vec{v}_{\mathbf{p} s}}{\left|\vec{v}_{\mathbf{p} s}\right|^{2}} \tau_{p^{\prime} s^{\prime}}^{\mathrm{tr}}
$$

Using explicitly the definition of $Q_{\mathbf{p} s}^{\mathbf{p}^{\prime} s^{\prime}}$ [Eq. (5)], we introduce the transport helicity index $\eta=s\left(\hat{v}_{\mathbf{p} s} \cdot \hat{p}\right) \equiv$ $\hat{z} \cdot\left(\vec{v}_{\mathbf{p} s} \times\langle\vec{\sigma}\rangle_{\mathbf{p} s}\right)= \pm 1$, which accounts for the reciprocal orientation of spin and velocity, and we recast Eq. (9) as follows:

$$
\frac{\tau_{\eta}^{\mathrm{tr}}}{\tau}=1+\frac{n_{i} v_{\mathrm{imp}}^{2}}{4 v_{F} \hbar^{2}} \sum_{\eta^{\prime}} \eta \eta^{\prime} p_{\eta^{\prime}} \tau_{\eta^{\prime}}^{\mathrm{tr}}
$$

where $v_{F}=\sqrt{2 m E_{F}}$ and $p_{\eta}=\left|m v_{F}-\eta p_{0}\right|$ are the Fermi momenta on the inner and outer Fermi surfaces. The above equation suggests that the index $\eta$ can be used to efficiently classify the states at the Fermi level across the different regimes. In particular, as illustrated in Fig. 1(a), where the value of $\eta$ is indicated by the red or blue colors of the surface, at $E_{F}>E_{0}, \eta$ simply coincides with $s$; on the contrary, at $E_{F}<E_{0}, \eta$ allows us to distinguish between the two Fermi circles that have the same value of $s$ but antiparallel quasiparticle velocities. Using this classification $\sigma_{\mathrm{dc}}^{B}$ can be cast as

$$
\sigma_{\mathrm{dc}}^{B}=\frac{e^{2} v_{F}}{4 \pi} \sum_{\eta} \tau_{\eta}^{\mathrm{tr}} p_{\eta}=\sum_{\eta} \sigma_{\eta},
$$

where the transport scattering times $\tau_{\eta}^{\mathrm{tr}}$ are given by the solution of Eq. (10), 


$$
\tau_{\eta}^{\mathrm{tr}}=\tau p_{\eta} / \bar{p}_{F},
$$

with $\bar{p}_{F}=1 / 2 \sum_{\eta} p_{\eta}$; i.e., $\bar{p}_{F}=m v_{F}$ for $E_{F}>E_{0}$ and $\bar{p}_{F}=p_{0}$ for $E_{F}<E_{0}$. As one can easily check, Eqs. (11) and (12) yield the Drude result Eq. (1) at $E_{F}>E_{0}$, and Eq. (2) in the DSO regime.

The physical relevance of $\eta$, as compared to the standard helicity $s$, is evident in Fig. 1(b), where we show that spin conservation forbids backscattering between states having the same value of $\eta$. What is more important, in the DSO regime the only allowed backscattering processes reverse the sign of the quasiparticle velocity without changing the direction of momentum. These effects determine the density dependence of the scattering times (a) and of the conductivities (b) of the majority $(\eta=-1)$ and minority $(\eta=+1)$ carriers shown in Fig. 2. As one can see in Fig. 2(b), transport is in general dominated by the majority carriers that, due to the suppression of backscattering, also have the larger transport scattering time, $\tau_{-}^{\mathrm{tr}}>\tau>\tau_{+}^{\mathrm{tr}}$. Let us focus on the transport properties of majority and minority carriers across the different regimes. In the HD regime $\tau$ is a constant and $\tau_{-}^{\text {tr }} / \tau$ increases as the density decreases due to the shrinking of the inner Fermi circle. At $n=n_{0}$, where only states with $\eta=-1$ are present, backscattering is completely suppressed and one recovers $\tau_{-}^{\mathrm{tr}}=2 \tau$, like, e.g., in graphene [46]. However, differently from graphene, as long as $n>n_{0}$, a compensation between SO effects on the velocity and on the transport scattering times of the two types of carriers restores the usual Drude conductivity, even for $n$ very close to $n_{0}$. This result is nontrivial: indeed, setting naively $\tau_{\eta}^{\mathrm{tr}}=\tau$ in Eq. (8) would lead to [42] $\sigma \simeq\left(n-n_{0} / 2\right) \tau_{0} / m$, i.e., $\sigma<\sigma_{\text {Drude }}$ even at $n>n_{0}$ [47]. On the other hand, as the system enters the

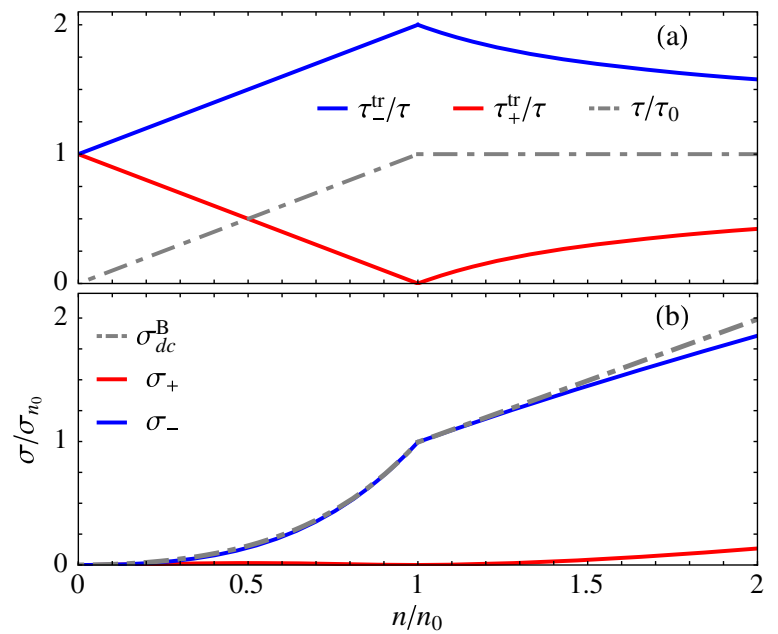

FIG. 2. (a) Density dependence of the ratio $\tau_{\mp} / \tau$ for the majority $\left(\tau_{-}^{\mathrm{tr}}\right.$, solid blue line $)$ and minority $\left(\tau_{+}^{\mathrm{tr}}\right.$, solid red line) carriers, and of the ratio $\tau / \tau_{0}$ (dashed line). (b) Density dependence of Boltzmann dc conductivity $\sigma_{\mathrm{dc}}^{B}$, and contribution $\sigma_{\mp}$ of the two types of carriers, in units of $\sigma_{n_{0}}=n_{0} e^{2} \tau_{0} / m$.
DSO regime $\tau$ starts to decrease linearly, as predicted by Eq. (6) and backscattering processes for the majority carriers are progressively restored. Both these effects quench $\tau_{-}^{\text {tr }}$ as $n<n_{0}$, see Fig. 2(a), leading to an overall sublinear behavior of the conductivity, see Fig. 2(b).

A deeper insight on dc transport comes from the generalization of the Boltzmann approach to fully include quantum effects. To this end, we use the Kubo linear response theory [48]. We start by noticing that, within the self-consistent Born approximation (SCBA), the retarded Green's function is diagonal in the helicity basis and it is given by the following matrix $[48,49]$,

$$
\left[G^{R}(\mathbf{p}, \omega)\right]_{s s^{\prime}}=g_{s}^{R}(p, \omega) \delta_{s s^{\prime}},
$$

where $g_{s}(p, \omega)=\left[\omega-E_{p}^{s}+E_{F}-\Sigma^{R}(\omega)\right]^{-1}$ denotes the Green function of electrons with helicity $s$. The self-energy $\Sigma^{R}(\omega)=n_{i} v_{\mathrm{imp}}^{2} /(2 \mathcal{V}) \sum_{\mathbf{p}, s} g_{s}^{R}(p, \omega)$ is spin and momentum independent [48]. At zero frequency its imaginary part defines the elastic scattering rate of quasiparticles, $\Gamma=-\operatorname{Im}\left[\Sigma^{R}(0)\right]=n_{i} v_{\mathrm{imp}}^{2} \pi /(2 \mathcal{V}) \sum_{\mathbf{p}, s} \mathcal{A}_{s}(p), \quad$ where $\mathcal{A}_{s}(p)=-(1 / \pi) \operatorname{Im} g_{s}^{R}(p, \omega=0)$ is the spectral function of each helicity band. In Fig. 3(a) we plot the numerical
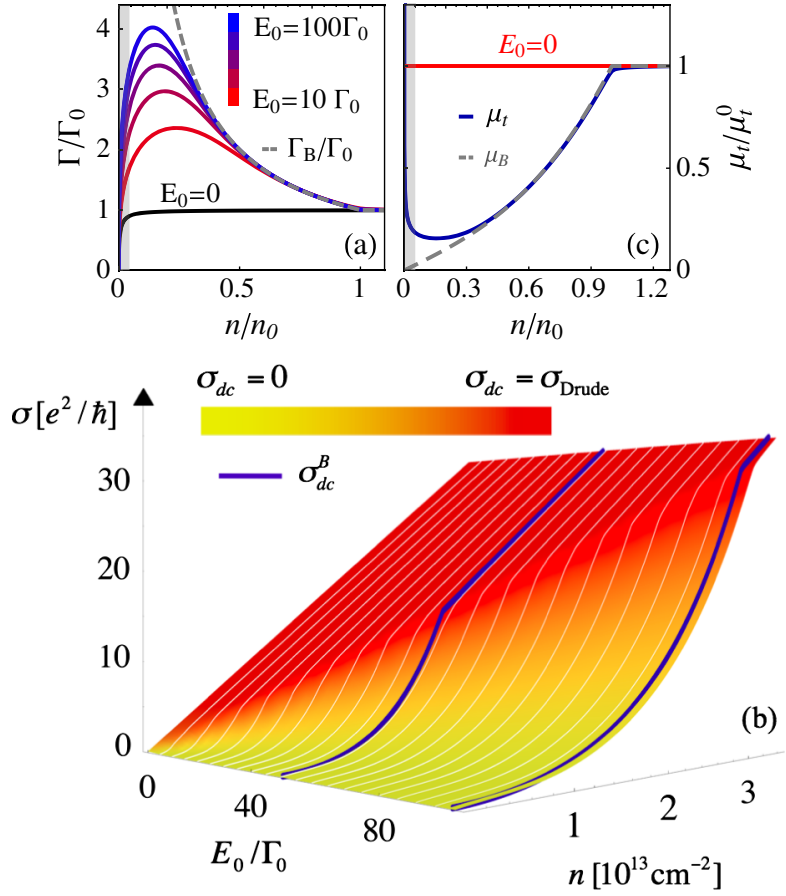

FIG. 3. (a) Scattering rate in units of $\Gamma_{0}$ as a function of $n / n_{0}$ for different $E_{0}$. The dashed line shows $\Gamma_{B}=1 / 2 \tau$ [Eq. (6)]. (b) Numerical dc conductivity [Eq. (14)] as a function of $E_{0} / \Gamma_{0}$ and $n$ for $\Gamma_{0}=0.5 \mathrm{meV}$ and $m=0.7 m_{e}$ (as appropriate, e.g., for $\mathrm{LaAlO}_{3} / \mathrm{SrTiO}_{3}$ interfaces [24]). The blue lines show Boltzmann conductivity for $E_{0}=50$ and $100 \Gamma_{0}$ (c) Mobility $\mu_{t}$ (solid line) normalized to $\mu_{t}^{0}=e /\left(2 \hbar \Gamma_{0} m\right)$, compared with Boltzmann's $\mu_{B}$ (dashed line). Shaded areas in (a) and (c) denote the low-density regions beyond the diffusive approximation. 
self-consistent $\Gamma$ as a function of the ratio $n / n_{0}$ for different values of the SO coupling and we compare it with the Boltzmann result $\Gamma_{B}=1 /(2 \tau)$. As expected, the Boltzmann result is accurate for large $n / n_{0}$, where $\Gamma \ll E_{F}$ holds and one can approximate the spectral function as $\mathcal{A}_{s}(p) \simeq \delta\left(E_{p}^{s}-E_{F}\right)$, and its accuracy increases with increasing $E_{0} / \Gamma_{0}$. On the contrary, as $E_{F}$ approaches the band edge, the DOS singularity is smeared by disorder and finite-band effects cut off the divergence of the Boltzmann result $\Gamma_{B}=\Gamma_{0} \sqrt{E_{0} / E_{F}}$ following from Eq. (6). In the extreme diluted limit [shaded region in Fig. 3(a)], $\Gamma$ as given by the SCBA vanishes and the diffusive approximation breaks down [42].

Within linear response theory the conductivity is given by the Kubo formula and, at $T=0$, it is related to the onshell current-current response function as follows [48],

$$
\sigma_{\mathrm{dc}}=\frac{\hbar}{2 \pi}\left(P_{x x}^{A R}-\operatorname{Re}\left[P_{x x}^{R R}\right]\right),
$$

where $P_{x x}^{L M}$ is given by

$$
P_{x x}^{L M}=\frac{1}{\mathcal{V}} \sum_{\mathbf{p}} \operatorname{Tr}\left[j_{x}(\mathbf{p}) G^{L}(\mathbf{p}, 0) J_{x}^{L M}(\mathbf{p}) G^{M}(\mathbf{p}, 0)\right],
$$

and the superscripts $L, M=A, R$ indicate advanced or retarded quantities. In the above equation $j_{x}(\mathbf{p})$ and $J_{x}^{L M}(\mathbf{p})$ denote the bare and dressed currents and they are, in general, represented by $2 \times 2$ matrices in the helicity space. In particular, $j_{x}(\mathbf{p})=e v_{x}$ is proportional to the bare velocity Eq. (7), while $J_{x}$ has to be determined selfconsistently [42] and it can be written as

$$
\left[\vec{J}^{L M}(\mathbf{p})\right]_{s s^{\prime}}=e\left[\vec{V}_{\mathbf{p} s}^{L M} \delta_{s s^{\prime}}-i \tilde{\alpha}^{L M} s\left(1-\delta_{s s^{\prime}}\right) \hat{t}_{p}\right],
$$

where $\vec{V}_{\mathbf{p} s}^{L M}=\mathbf{p} / m+s \tilde{\alpha}^{L M} \hat{p}$ denotes the dressed quasiparticle velocity. By comparing Eqs. (7) and (16) one sees that, as usual [48] in the Kubo formalism, the effects of scattering by impurities are encoded, via the vertex function $\tilde{\alpha}^{L M}$, in the renormalization of the velocity. As we show below, under appropriate conditions, these effects are equivalently accounted for in Boltzmann language by the transport scattering times.

The anomalous velocity $\tilde{\alpha}^{L M}$ also plays an important role in the spin-Hall effect [50]. In this context it was shown that, although $\tilde{\alpha}^{R A}=0$ in the HD regime $[36,51,52]$ and $\tilde{\alpha}^{R A} \neq 0$ in the DSO regime [49], in both regimes the spinHall conductivity vanishes. This result follows straightforwardly from the vanishing of $\tilde{\alpha}^{R A}$ in the HD regime while it can be proven by an explicit calculation in the DSO regime [49].

Using Eqs. (7) and (16), the current response function Eq. (15) can be cast as the sum of inter- and intraband terms: $P_{x x}^{L M}=P_{\text {intra }}^{L M}+P_{\text {inter }}^{L M}$, where

$$
\begin{gathered}
P_{\text {intra }}^{L M}=\frac{e^{2}}{2 \mathcal{V}} \sum_{\mathbf{p s}} \vec{v}_{\mathbf{p} s} \cdot \overrightarrow{\mathrm{V}}_{\mathbf{p} s}^{L M} g_{s}^{L}(p, 0) g_{s}^{M}(p, 0), \\
P_{\text {inter }}^{L M}=\frac{e^{2}}{2 \mathcal{V}} \alpha \tilde{\alpha}^{L M} \sum_{\mathbf{p} s \neq s^{\prime}} g_{s}^{L}(p, 0) g_{s^{\prime}}^{M}(p, 0) .
\end{gathered}
$$

From a numerical self-consistent solution of the self-energy and vertex equations, we calculate the fully quantum dc conductivity Eq. (14). The results are shown in Fig. 3(b), where we plot the conductivity as a function of the electronic density and of the SO coupling. Here we also plot the Boltzmnann conductivity (blue lines) for two values of $E_{0}$, showing that Kubo results follow quite closely the Boltzmann prediction.

The equivalence between the two approaches can be proven in the limit of vanishingly small broadening of the spectral functions, $\Gamma \ll E_{F}$, where we can discard [48] the $R R$ term in Eq. (14). Indeed, by also neglecting the interband contribution to $P^{R A}$, relevant only at $n \simeq n_{0}$ where the spectral functions of the two chiral bands overlap, we can recast the conductivity as

$$
\sigma_{\mathrm{dc}} \simeq \frac{e^{2}}{4 \mathcal{V} \Gamma} \sum_{\mathbf{p s}} \vec{v}_{\mathbf{p} s} \cdot \vec{V}_{\mathbf{p} s}^{R A} \delta\left(E_{F}-E_{p}^{s}\right),
$$

which, by direct comparison with Eq. (8), yields $\sigma_{\mathrm{dc}} \simeq \sigma_{\mathrm{dc}}^{B}$ provided that $\vec{V}_{\mathbf{p} s}^{R A} \simeq \tau_{\mathbf{p} s}^{t r} \vec{v}_{\mathbf{p} s} / \tau$. In the limit $\Gamma \ll E_{F}$ this relation is a straightforward consequence of the vanishing of $\tilde{\alpha}^{R A}$ for $E_{F}>E_{0}$, and it can be easily proved for $E_{F}<E_{0}$ using $\tilde{\alpha}^{R A} \simeq \alpha\left(1-E_{F} / E_{0}\right)$ [42]. This shows, in particular, that on the Fermi circles $V_{\mathbf{p} s} / v_{F}=\tau_{\eta}^{\mathrm{tr}} / \tau$.

The deviations between Boltzmann and Kubo results are better seen in Fig. 3(c), where we compare the corresponding mobilities, respectively defined as $\mu_{B}=\sigma_{\mathrm{dc}}^{B} /(e n)$ and $\mu_{t}=\sigma_{\mathrm{dc}} /(e n)$, and they can be ultimately ascribed to two factors. First, finite-band effects, that are mostly relevant for $n \lesssim 0.3 n_{0}$ and are responsible for the deviations of $\Gamma$ from $\Gamma_{B}$, shown in Fig. 3(a), and for the relevance of $R R$ terms [42] that in turn imply that for small densities $\mu_{t}$ tends to saturate, in contrast to $\mu_{B}$. Second, interband terms that are mostly relevant at $n \simeq n_{0}$ and give a smoothening of dependence of $\mu_{t}$ on $n$. This effect could also be captured by replacing the semiclassical Boltzmann equation with a fully quantum kinetic equation that also includes the offdiagonal components of the velocity operator Eq. (7) and of the nonequilibrium density matrix in the helicity space. This allows one to account, in the presence of external fields, for the coherent superpositions of states with different helicities, as explained, e.g., in Refs. [33,53-55].

In conclusion, we have shown that in Rashba 2DEG SO coupling entails an unconventional dc conductivity, strongly dependent on the density and on the SO coupling strength. The experimental verification of our results 
requires the condition for diffusive transport $\left(E_{F} \gg \Gamma\right)$ to be fulfilled in the DSO regime $\left(E_{0}>E_{F}\right)$ : this ultimately implies $E_{0}>E_{F} \gg \Gamma$. The conductivity anomalies in the DSO regime can then be accessible experimentally in relatively clean $\left(\Gamma_{0} \sim 1 \mathrm{meV}\right)$ samples of the strong-Rashba materials mentioned in the introduction (see Refs. [3-32]), where $E_{0} \approx$ $10-140 \mathrm{meV}$ and $m \approx 0.2-0.7 m_{e}$, corresponding to electron densities $n_{0} \approx(0.6-8) \times 10^{13} \mathrm{~cm}^{-2}$ [56]. Finally, we also remark that the large value of $E_{0}$ in these systems guarantees that our zero-temperature results will provide a good description for real materials up to temperature scales $k_{B} T \sim E_{0}$. In addition, the occurrence of the DSO anomalies at relatively large densities $n \simeq n_{0}$ also justifies neglecting the electronelectron interactions, even though a full understanding of the conductivity anomalies in the diluted regime where interactions become relevant is certainly an interesting topic for future investigation.

We gratefully acknowledge fruitful discussions with S. Caprara, C. Castellani, M. Grilli, and R. Raimondi. We acknowledge financial support by Italian MIUR under projects FIRB-HybridNanoDev-RBFR1236VV, PRIN-RIDEIRON-2012X3YFZ2, Premiali-2012 ABNANOTECH, and by the European project FP7PEOPLE-2013-CIG "LSIE_2D.”

[1] D. D. Awschalom and M. J. Flatté, Nat. Phys. 3, 153 (2007).

[2] A. Manchon, H. C. Koo, J. Nitta, S. M. Frolov, and R. A. Duine, Nat. Mater. 14, 871 (2015).

[3] C. R. Ast, J. Henk, A. Ernst, L. Moreschini, M. C. Falub, D. Pacilé, P. Bruno, K. Kern, and M. Grioni, Phys. Rev. Lett. 98, 186807 (2007)

[4] C. R. Ast, D. Pacilé, L. Moreschini, M. C. Falub, M. Papagno, K. Kern, M. Grioni, J. Henk, A. Ernst, S. Ostanin, and P. Bruno, Phys. Rev. B 77, 081407 (2008).

[5] I. Gierz, T. Suzuki, E. Frantzeskakis, S. Pons, S. Ostanin, A. Ernst, J. Henk, M. Grioni, K. Kern, and C. R. Ast, Phys. Rev. Lett. 103, 046803 (2009).

[6] H. Mirhosseini, A. Ernst, S. Ostanin, and J. Henk, J. Phys. Condens. Matter 22, 385501 (2010).

[7] K. Yaji, Y. Ohtsubo, S. Hatta, H. Okuyama, K. Miyamoto, T. Okuda, A. Kimura, H. Namatame, M. Taniguchi, and T. Aruga, Nat. Commun. 1, 17 (2010).

[8] D. V. Gruznev, L. V. Bondarenko, A. V. Matetskiy, A. A. Yakovlev, A. Y. Tupchaya, S. V. Eremeev, E. V. Chulkov, J.-P. Chou, C.-M. Wei, M.-Y. Lai, Y.-L. Wang, A. V. Zotov, and A. A. Saranin, Sci. Rep. 4, 4742 (2014).

[9] J. C. Rojas Sánchez, L. Vila, G. Desfonds, S. Gambarelli, J. P. Attané, J. M. De Teresa, C. Magén, and A. Fert, Nat. Commun. 42944 (2013).

[10] S. V. Eremeev, I. A. Nechaev, Y. M. Koroteev, P. M. Echenique, and E. V. Chulkov, Phys. Rev. Lett. 108, 246802 (2012).

[11] M. Bahramy, B.-J. Yang, R. Arita, and N. Nagaosa, Nat. Commun. 3, 679 (2012).

[12] M. Sakano, M. S. Bahramy, A. Katayama, T. Shimojima, H. Murakawa, Y. Kaneko, W. Malaeb, S. Shin, K. Ono,
H. Kumigashira, R. Arita, N. Nagaosa, H. Y. Hwang, Y. Tokura, and K. Ishizaka, Phys. Rev. Lett. 110, 107204 (2013).

[13] X. Xi, C. Ma, Z. Liu, Z. Chen, W. Ku, H. Berger, C. Martin, D. B. Tanner, and G. L. Carr, Phys. Rev. Lett. 111, 155701 (2013).

[14] Y. L. Chen, M. Kanou, Z. K. Liu, H. J. Zhang, J. A. Sobota, D. Leuenberger, S. K. Mo, B. Zhou, S.-L. Yang, P. S. Kirchmann, D. H. Lu, R. G. Moore, Z. Hussain, Z. X. Shen, X. L. Qi, and T. Sasagawa, Nat. Phys. 9, 704 (2013).

[15] L. Ye, J. G. Checkelsky, F. Kagawa, and Y. Tokura, Phys. Rev. B 91201104 (2015).

[16] F.-X. Xiang, X.-L. Wang, M. Veldhorst, S.-X. Dou, and M. S. Fuhrer, Phys. Rev. B 92, 035123 (2015).

[17] Y. Gui, C. Becker, N. Dai, J. Liu, Z. Qiu, E. Novik, M. Schaefer, X. Shu, J. Chu, H. Buhmann et al., Phys. Rev. B 70, 115328 (2004).

[18] A. Ohtomo and H. Y. Hwang, Nature (London) 427, 423 (2004).

[19] N. Reyren, S. Thiel, A. D. Caviglia, L. F. Kourkoutis, G. Hammerl, C. Richter, C. W. Schneider, T. Kopp, A.-S. Ruetschi, D. Jaccard et al., Science 317, 1196 (2007).

[20] A. D. Caviglia, S. Gariglio, N. Reyren, D. Jaccard, T. Schneider, M. Gabay, S. Thiel, G. Hammerl, J. Mannhart, and J.-M. Triscone, Nature (London) 456, 624 (2008).

[21] C. Bell, S. Harashima, Y. Kozuka, M. Kim, B. G. Kim, Y. Hikita, and H. Y. Hwang, Phys. Rev. Lett. 103, 226802 (2009).

[22] A. D. Caviglia, M. Gabay, S. Gariglio, N. Reyren, C. Cancellieri, and J.-M. Triscone, Phys. Rev. Lett. 104, 126803 (2010).

[23] M. Ben Shalom, M. Sachs, D. Rakhmilevitch, A. Palevski, and Y. Dagan, Phys. Rev. Lett. 104, 126802 (2010).

[24] J. Biscaras, N. Bergeal, S. Hurand, C. Grossetete, A. Rastogi, R. C. Budhani, D. LeBoeuf, C. Proust, and J. Lesueur, Phys. Rev. Lett. 108, 247004 (2012).

[25] S. Seri, M. Schultz, and L. Klein, Phys. Rev. B 86, 085118 (2012).

[26] A. Joshua, J. Ruhman, S. Pecker, E. Altman, and S. Ilani, Proc. Natl. Acad. Sci. U.S.A. 110, 9633 (2013).

[27] S. Hurand, A. Jouan, C. Feuillet-Palma, G. Singh, J. Biscaras, E. Lesne, N. Reyren, A. Barthlmy, M. Bibes, J. E. Villegas, C. Ulysse, X. Lafosse, M. Pannetier-Lecoeur, S. Caprara, M. Grilli, J. Lesueur, and N. Bergeal, Sci. Rep. 5 12751 (2015).

[28] Q. Liu, Y. Guo, and A. J. Freeman, Nano Lett. 13, 5264 (2013).

[29] J. Biscaras, S. Hurand, C. Feuillet-Palma, A. Rastogi, R. C. Budhani, N. Reyren, E. Lesne, J. Lesueur, and N. Bergeal, Sci. Rep. 4, 6788 (2014).

[30] Z. Zhong, L. Si, Q. Zhang, W.-G. Yin, S. Yunoki, and K. Held, Adv. Mater. Interfaces 2, 1400445 (2015).

[31] Z. Zhong, A. Tóth, and K. Held, Phys. Rev. B 87161102 (2013).

[32] A. Joshua, S. Pecker, J. Ruhman, E. Altman, and S. Ilani, Nat. Commun. 3, 1129 (2012).

[33] A. V. Khaetskii, Phys. Rev. Lett. 96, 056602 (2006).

[34] R. Raimondi, M. Leadbeater, P. Schwab, E. Caroti, and C. Castellani, Phys. Rev. B 64, 235110 (2001).

[35] P. Schwab and R. Raimondi, Eur. Phys. J. B 25, 483 (2002). 
[36] R. Raimondi and P. Schwab, Phys. Rev. B 71, 033311 (2005).

[37] A. Agarwal, S. Chesi, T. Jungwirth, J. Sinova, G. Vignale, and M. Polini, Phys. Rev. B 83, 115135 (2011).

[38] D. Bercioux and P. Lucignano, Rep. Prog. Phys. 78, 106001 (2015).

[39] Y. A Bychkov and E. I. Rashba, Pis'ma Zh. Eksp. Teor. Fiz. 39, 66 (1984) [JETP Lett. 39, 78 (1984)].

[40] E. Cappelluti, C. Grimaldi, and F. Marsiglio, Phys. Rev. Lett. 98, 167002 (2007).

[41] J. M. Ziman, Principles of the Theory of Solids (Cambridge University Press, Cambridge, England, 1972).

[42] See Supplemental Material at http://link.aps.org/ supplemental/10.1103/PhysRevLett.116.166602 for more details, which includes Refs. [43-45].

[43] H. Bruus and C. Flenshberg, Many-Body Quantum Theory in Condensed Matter Physics (Oxford University Press, New York, 2004).

[44] A. Knigavko and J. P. Carbotte, Phys. Rev. B 72, 035125 (2005).

[45] N. A. Sinitsyn, A. H. MacDonald, T. Jungwirth, V. K. Dugaev, and J. Sinova, Phys. Rev. B 75, 045315 (2007).

[46] See, e.g., A. H. Castro Neto, F. Guinea, N. M. R. Peres, K. S. Novoselov, and A. K. Geim, Rev. Mod. Phys. 81, 109 (2009), and references therein.

[47] Z. Li, F. Marsiglio, and J. P. Carbotte, Sci. Rep. 3, 2828 (2013).
[48] G. D. Mahan, Many-Particle Physics (Springer, Berlin, 2000).

[49] C. Grimaldi, E. Cappelluti, and F. Marsiglio, Phys. Rev. B 73, 081303 (2006).

[50] M. Dyakonov and V. I. Perel, Pis'ma Zh. Eksp. Teor. Fiz. 13, 467 (1971).[Sov. Phys. JETP 33, 1053 (1971)].

[51] R. Raimondi, P. Schwab, C. Gorini, and G. Vignale, Ann. Phys. (Amsterdam) 524, 153 (2011).

[52] G. Vignale, J. Supercond. Novel Magn. 23, 3 (2010).

[53] M. I. D'yakonov and A. V. Khaetskii, Zh. Eksp. Teor. Fiz. 86, 1843 (1984).[M. I. D'yakonov and A. V. KhaetskiiSov. Phys. JETP 59, 1072 (1984)].

[54] A. V. Shytov, E. G. Mishchenko, H.-A. Engel, and B. I. Halperin, Phys. Rev. B 73, 075316 (2006).

[55] R. Raimondi, C. Gorini, P. Schwab, and M. Dzierzawa, Phys. Rev. B 74, 035340 (2006).

[56] A concrete example is given in Fig. 3 where we chose parameter values [27] appropriate for $\mathrm{LaAlO}_{3} / \mathrm{SrTiO}_{3}$ interfaces. An even more favorable situation is found on the surface of BiTeI where $E_{0} \approx 0.1 \mathrm{eV}$ [12], and the electron mass $m \sim 0.2 m_{e}$ [13], leading to $n_{0}=1.9 \times 10^{13} \mathrm{~cm}^{-2}$. Similarly, in surface alloys (see, e.g., Ref. [5]), $E_{0}$ and $m$ are approximately $140 \mathrm{meV}$ and $0.7 m_{e}$, predicting a DSO regime for $n \leq n_{0} \sim 8 \times 10^{13} \mathrm{~cm}^{-2}$. We remark that both in surface alloys [4] and in bulk BiTeI [16] the regime $E_{F} \lesssim E_{0}$ has already been demonstrated to be accessible experimentally. 